\title{
Association of Interictal Epileptiform Discharges with Sleep and Anti-Epileptic Drugs
}

\author{
Latika Mohan Jayvardhan Singh Yogesh Singh Rajesh Kathrotia Arun Goel
}

Department of Physiology, All India Institute of Medical Sciences, Rishikesh, India

\author{
Key Words \\ Anti-epileptic drugs · Interictal epileptiform discharges . \\ Electroencephalogram · Seizures
}

\begin{abstract}
Background: The presence of interictal epileptiform discharges (IEDs) in electroencephalogram (EEG) is diagnostic of epilepsy. Latent IEDs are activated during sleep. Anti-epileptic drugs (AEDs) improve sleep. AEDs, sleep, and IEDs may interact and affect epilepsy management. Purpose: To explore the occurrence of IEDs and its association with sleep and AED status in suspected patients of epilepsy. Methods: EEG records were collected of suspected patients of epilepsy who reported to the electrophysiology laboratory of a tertiary care hospital during 1 year. The anthropometric details, clinical presentations, and AED status of the patients were recorded from the EEG records. Patients were divided into 2 categories based on whether AEDs had been started prior to the EEG evaluation (category-I) or not (category-II). The occurrences of IEDs in EEG recordings in both categories were analyzed. Results: In 1 year, 138 patients were referred for diagnostic EEG evaluation. One-hundred-two patients fulfilled the inclusion criteria, of which 57 patients (53\%) belonged to category-I and 45 patients (47\%) belonged to category-II. Incidence of IEDs, suggestive of definite diagnosis of epilepsy in category-I was $88 \%$ and in category-II was $69 \%$, and this difference was statistically significant ( $p=0.02$ ). Conclusion: The increased proportion of IEDs in category-I
\end{abstract}

\section{KARGER}

E-Mail karger@karger.com

www.karger.com/aon patients may be due to high clinical suspicion or compounding interaction of AEDs and sleep. More extensive studies are required to delineate the complex interaction of AEDs, sleep, and IEDs so that judicious yet prompt management of epilepsy can be carried out.

(c) 2016 S. Karger AG, Basel

\section{Introduction}

Epilepsy is a common neurological disorder affecting approximately 50 million people worldwide and $80 \%$ of these patients live in developing countries [1]. Clinically, epilepsy is defined as a minimum of 2 or more unprovoked seizures [2]. Seizure, a paroxysmal event due to abnormal hyper-synchronous neuronal discharge, can have varied neurological manifestations according to its site of origin and spread [3]. Studies have shown that $70 \%$ of children and adults with epilepsy can be successfully treated with anti-epileptic drugs (AEDs) [1].

Electroencephalogram (EEG) is a gold standard investigation in the diagnosis and management of epilepsy and is routinely warranted. The presence, frequency, and distribution pattern of interictal epileptiform discharges (IEDs) in EEG are suggestive of epilepsy [4]. IEDs, which are sub-clinical events picked up during EEG, are rarely seen in persons without history of seizures [5]. EEG performed during sleep improves the diagnostic and prognostic values of EEG in suspected epilepsy patients.
C 2016 S. Karger AG, Basel

0972-7531/16/0234-0230\$39.50/0 
Literature suggest that latent IEDs are mainly activated during non-rapid-eye-movement (NREM) stages I and II sleep and focal IEDs tend to become bilateral and more generalized [5-8]. Thus, EEG performed during sleep is a standard procedure in the diagnosis of epilepsy.

AEDs are also known to alter the sleep architecture, resulting in daytime sleepiness [9-11]. Therefore, it is plausible to postulate that AEDs may directly and indirectly influence IEDs. Therefore, this interdependent complex dynamics of AEDs, sleep, and IEDs need to be explored.

In this study, we investigated the occurrence of IEDs and its interaction with sleep and AED status in suspected patients of seizure disorder. This research may help in providing evidence-based guideline for the management of epilepsy.

\section{Methods}

Study Design

A selective hospital record-based retrospective study.

\section{Study Population}

EEG records were collected of all patients who were referred to the EEG laboratory of a tertiary care hospital for confirmatory diagnosis of suspected seizure disorder between July 2014 and June 2015.

\section{EEG Protocol}

The patients were instructed for overnight sleep deprivation prior to the day of EEG investigation. EEG was recorded in a dark, silent room for approximately $45 \mathrm{~min}$ on a 24 -channel softwarebased computerized digital EEG (RMS, 32 Superspec). The electrodes were placed according to the international 10-20 system and impedance values were kept below 5 kilo-ohms. Intermittent photic stimulation, hyperventilation, and sleep were selected as activation procedures. The chronological sequence of recording were basal eye-open and eye-close record ( $2 \mathrm{~min}$ each) followed by hyperventilation (3 min), intermittent photic stimulation ( $3 \mathrm{~min}$ ), and finally sleep record (35 min). Visual analysis and reporting of EEG was performed on the 16-channel Monopolar2 (R) montages (FP2-A2, F4-A2, C4-A2, P4-A2, FP1-A1, F3-A1, C3-A1, P3-A1, F8-A2, T4-A2, T6-A2, O2-A2, F7-A1, T3-A1, T5-A1 and O1-A1) by one expert electro-physiologist who was blinded about the clinical history of patients during analysis.

\section{Data Parameters}

From the records, we noted patient details, clinical presentations, and AED status of patients. Frequency (e.g., solitary or multiple) and distribution patterns (e.g., focal or generalized) of IEDs were also noted.

\section{Selection Criteria of the Study}

We considered those records where complete EEG protocol was followed. Incomplete records and records of patients who required sedation with chloral hydrate were excluded. Based on their
Table 1. Characteristics of patients at presentation

\begin{tabular}{lc}
\hline Characteristics & $\mathrm{n}=138$ \\
\hline Age, years, median (range) & $19.5(1-76)$ \\
Gender & \\
$\quad$ Male & 83 \\
$\quad$ Female & 55 \\
Cases & \\
$\quad$ New & 101 \\
$\quad$ Relapse & 37 \\
AEDs prescribed & 73 \\
Single drug & 51 \\
Multiple drugs & 22 \\
Duration of AED use, days, median (range) & $20(1-82)$ \\
Sufficient sleep EEG recorded & 102 \\
Pattern of EEG report & 102 \\
$\quad$ IEDs & 81 \\
$\quad$ Normal & 21 \\
\hline
\end{tabular}

AED status at the time of referral of EEG, we divided the selected population into 2 categories. Category- 1 consisted of patients who were prescribed AEDs based on strong clinical suspicion and category-II patients were not prescribed AEDs prior to diagnostic EEG.

\section{Statistical Analysis}

Statistical analysis was performed using SPSS software version 20. Difference in proportions of IEDs was calculated in both the categories by chi-square test and a $\mathrm{p}$ value $<0.05$ was considered significant.

\section{Results}

In the specified duration of study, a total of $138 \mathrm{pa}-$ tients suspected of epilepsy/seizure disorder were referred for EEG evaluation. The referral departments were pediatrics $(n=40)$, neurosurgery $(n=39)$, psychiatry $(n=$ $37)$, and general medicine $(n=22)$. Sixty percent of patients were males and $40 \%$ were females. Median age of patients was 19.5 (range 1-76) years. Twenty-seven percent patients were known cases of epilepsy with relapse, whereas $73 \%$ patients presented for the first time with complaints suggestive of epilepsy/seizure disorder (table 1). Only 102 (74\%) patients fulfilled the selection criteria of our study. Detailed description of the selection criteria is shown in figure 1.

Out of 102 patients who fulfilled our study criteria (completed EEG protocol without using sedation), 53\% patients had been on AEDs prior to EEG (category-I) while $47 \%$ patients belonged to category-II. The proportion of IED records in category-I was $88 \%$ and category-II 
Fig. 1. Flow chart of selection criteria.

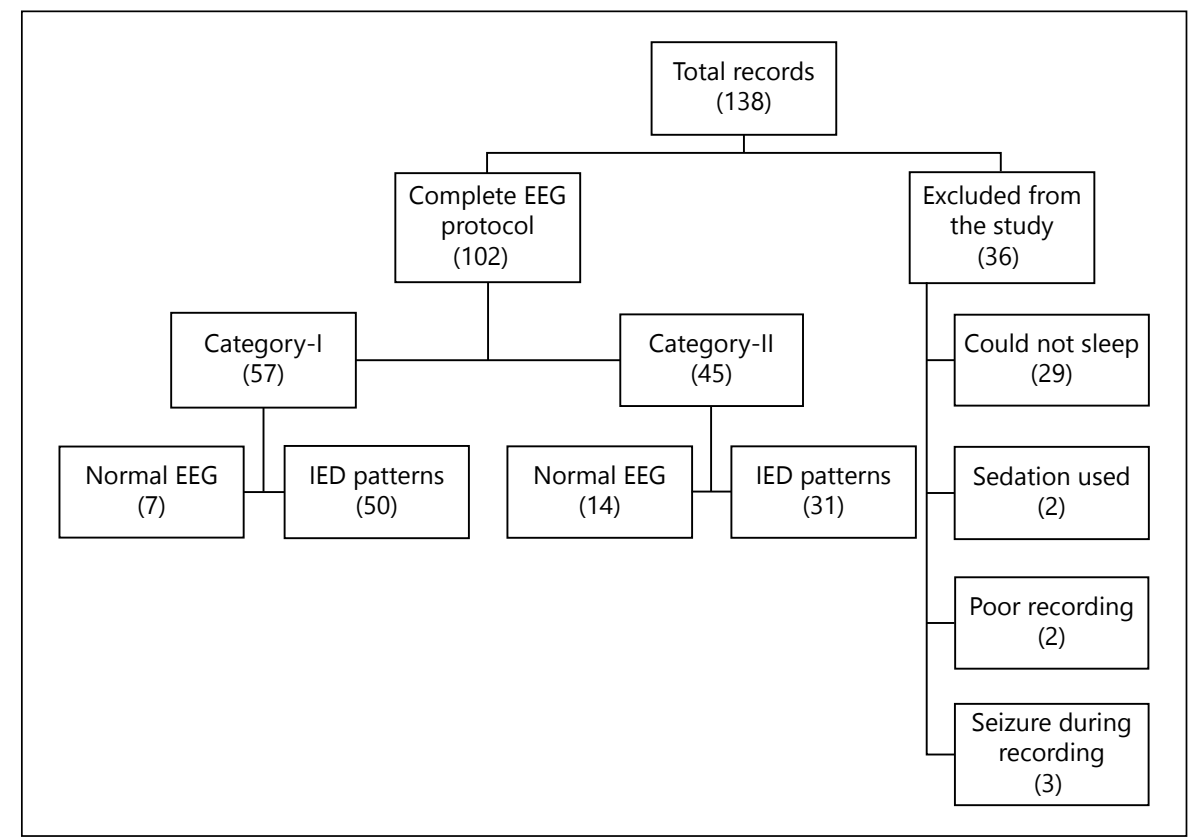

Fig. 2. IED patterns.

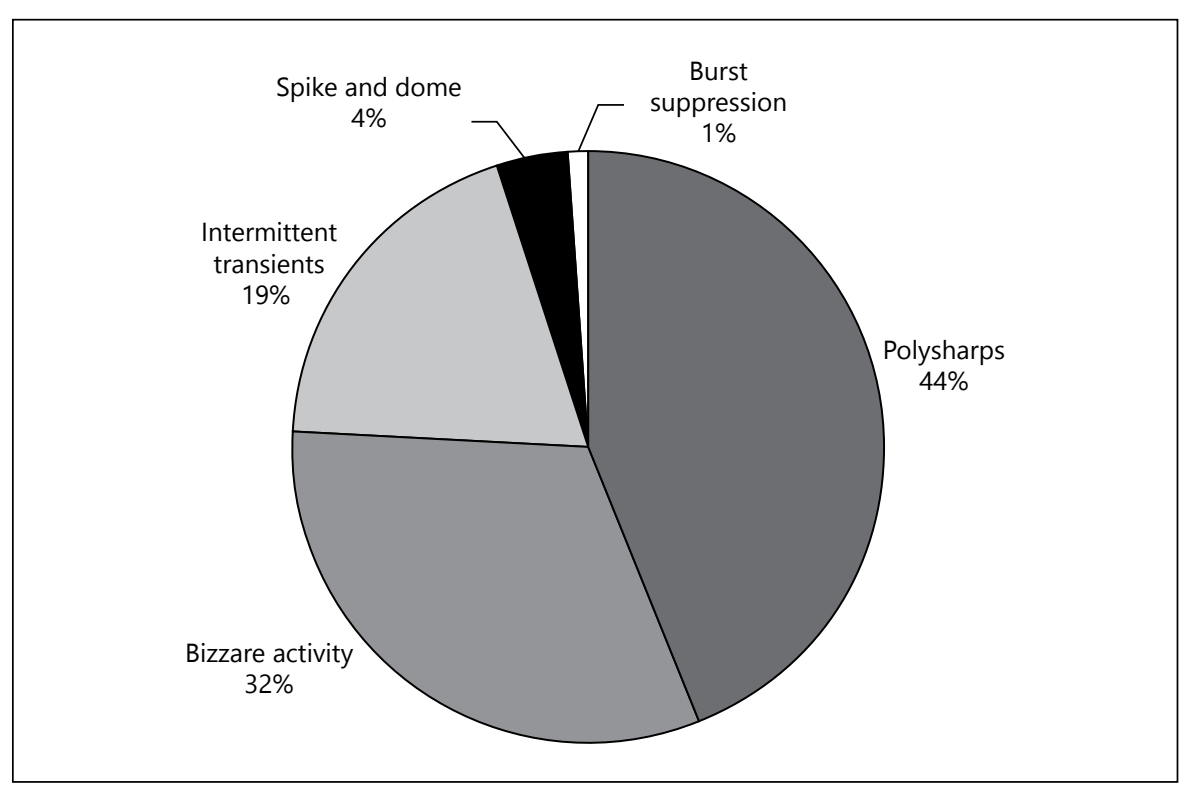

was $69 \%$, and this difference in proportion was statistically significant $(\mathrm{p}=0.02)$. The qualitative description of types [12] of IEDs is shown in figure 2.

The mean duration of treatment in category-I patients was 20 days (range $1-82$ days), and $70 \%$ patients were on single drug and $30 \%$ patients on multiple drugs. However, the association between duration of AEDs and IED in category-I could not be quantified because of the short duration and heterogeneous treatment (table 2).
Table 2. Relative proportion of subjects having IEDs with or without AEDs

\begin{tabular}{|c|c|c|c|c|}
\hline \multicolumn{3}{|c|}{ Sleep EEG $(n=102)$} & \multirow[t]{3}{*}{ Chi square } & \multirow[t]{3}{*}{$\mathrm{p}$ value } \\
\hline \multirow[t]{2}{*}{ AEDs } & \multicolumn{2}{|c|}{ IEDs, n (\%) } & & \\
\hline & present & absent & & \\
\hline Yes & $50(87.7)$ & $7(12.3)$ & \multirow[t]{2}{*}{5.45} & \multirow[t]{2}{*}{0.02} \\
\hline No & $31(68.9)$ & $14(31.1)$ & & \\
\hline
\end{tabular}




\section{Discussion}

Activation procedures are routinely employed during EEG to detect the latent IEDs. Sleep deprivation, hyperventilation, and intermittent photic stimulation are common activation procedures. The sequence of these activation procedures may have an effect on the induction of sleep in the laboratory settings [13]. In our study, 74\% patients could fall asleep (up to NREM stage II) during the EEG in laboratory. Reported percentage of sleep during EEG varied between 22 and 57\% [14]. In our setting, the high proportion of patients falling asleep in the EEG laboratory could be because of the sequence of activation procedure where sleep was the last one in the sequence, and the second possibility was strict adherence to overnight sleep deprivation before the EEG procedure.

We found statistically significant increase in the incidence of IEDs in category-I patients as compared to category-II patients, which may be because of 2 possibilities. The first obvious possible reason is the high clinical suspicion by the referring physician, reflecting a high probability of having epilepsy, and the second reason is the compounding effect of AEDs with sleep and IEDs.

AEDs were started in category-I patients before EEG referral and the median duration of treatment with AEDs in these patients was 20 days. In-vitro mice brain model studies have shown that interictal activity is unaffected by AEDs at levels that are effective to stop seizures [15]. However, in general, AEDs improve sleep architecture, decrease sleep latency and nocturnal awakening which indirectly may modify IEDs [9-11].

Sleep is an activation procedure for the detection of latent IEDs. Improved sleep architecture, mainly NREM stages I and II, may increase the frequency of IEDs and may also affect IED distribution pattern [5-8]. The suggested mechanism of NREM sleep to activate IEDs is the synchronization of neuronal assembly between brainstem, thalamus, and cortex [16-19].

Therefore, in category-I patients AEDs may indirectly increase the IEDs through its effect on sleep as the direct effect of AEDs used to control the seizure has shown no effect on IEDs. This increase in IEDs due to AEDs may contribute to some false positive reporting. Detection of these types of false positive IEDs due to activation procedure by sleep should be interpreted with caution. However, the prolonged treatment with AEDs may cause absence of IEDs by direct stabilization of neural membrane permeability, which could not be established in the present study because of short dura- tion of treatment. The true incidence of IEDs and epilepsy in high-risk patients without the compounding effect of AEDs can be assessed by prospective study where both case controls are selected from the high-risk group and controls are deprived of AEDs in spite of high clinical suspicion. However, it will not be ethical to conduct such prospective study and randomly assign treatment to one group and deprive treatment to the other group.

IEDs are rarely seen in healthy persons [4]. The presence of IEDs and its frequency and distribution is used to diagnose, classify, and mange epilepsy [20]. Thus, the presence of few false positive IEDs in high-risk patients may interfere with the management of epilepsy. Therefore, authors recommend that in high-risk patients, EEG should be done as early as in the out-patient setting before starting AEDs.

Few limitations were inherent in the present study, for example, both the patient categories were selected retrospectively. Additionally, visual interpretations of EEG have their own limitations and subjective biases. Future prospective studies with larger sample size need to be designed to substantiate the preliminary findings and indications of the present study.

\section{Authorship Contributions}

L.M. contributed to the design of study, critical revision of draft and valuable inputs. J.S. contributed to data collection, drafting of manuscript, interpretation of results. Y.S. contributed to data collection, interpretation of results R.K. contributed to drafting of manuscript, statistical analysis, interpretation of results and A.G. involved in design and interpretation of study.

\section{Disclosure Statements}

The authors declare no conflict of interest. This study received no funding from any agency. The manuscript is in compliance with International Committee of Medical Journal Editor's guidelines.

References

Ann Neurosci 2016;23:230-234

1 WHO: Epilepsy. http://www.who.int/ mediacentre/factsheets/fs999/en/ (accessed December 1, 2015).

2 Kasper DL, Braunwald E, Fauci AS, Hauser SL, Longo DL, Jameson JL, Loscalzo J: Harrison's Principles of Internal Medicine, ed 17. New York, McGraw-Hill Medical Publishing Division, 2008.

3 Jan MM: Clinical review of pediatric epilepsy. Neurosciences (Riyadh) 2005;10:255-264. 
4 So EL: Interictal epileptiform discharges in persons without a history of seizures: what do they mean? J Clin Neurophysiol 2010;27:229238.

5 Dinner DS: Effect of sleep on epilepsy. J Clin Neurophysiol 2002;19:504-513.

6 Sammaritano M, Gigli GL, Gotman J: Interictal spiking during wakefulness and sleep and the localization of foci in temporal lobe epilepsy. Neurology 1991;41(2 pt 1):290297.

7 Malow BA, Lin X, Kushwaha R, Aldrich MS: Interictal spiking increases with sleep depth in temporal lobe epilepsy. Epilepsia 1998;39: 1309-1316.

8 Malow BA, Selwa LM, Ross D, Aldrich MS: Lateralizing value of interictal spikes on overnight sleep-EEG studies in temporal lobe epilepsy. Epilepsia 1999;40:1587-1592.

9 Sammaritano M, Sherwin A: Effect of anticonvulsants on sleep. Neurology 2000;54(5 suppl 1): S16-S24.
10 Placidi F, Scalise A, Marciani MG, Romigi A, Diomedi M, Gigli GL: Effect of antiepileptic drugs on sleep. Clin Neurophysiol 2000; 111(suppl 2):S115-S119.

11 Jain SV, Glauser TA: Effects of epilepsy treatments on sleep architecture and daytime sleepiness: an evidence-based review of objective sleep metrics. Epilepsia 2014;55:26-37.

12 Latika M, Nitu R, Yogesh S: Assessment of EEG as a diagnostic and prognostic indicator tool in the febrile seizures. Indian J Physiol Pharmacol 2015;59:251-260.

13 Kaleyias J, Kothare SV, Pelkey M, Harrison G, Legido A, Khurana DS: Achieving sleep state during EEG in children; sequence of activation procedures. Clin Neurophysiol 2006;117: 1582-1584.

14 Gilbert DL, DeRoos S, Bare MA: Does sleep or sleep deprivation increase epileptiform discharges in pediatric electroencephalograms? Pediatrics 2004;114:658-662.
15 D’Antuono M, Köhling R, Ricalzone S, Gotman J, Biagini G, Avoli M: Antiepileptic drugs abolish ictal but not interictal epileptiform discharges in vitro. Epilepsia 2010;51:423-431.

16 Guerrini R, Pellacani S: Benign childhood focal epilepsies. Epilepsia 2012;53(suppl 4):918.

17 Samaitienè R, Norkūnienè J, Tumienè B, Grikinienė J: Sleep and behavioral problems in rolandic epilepsy. Pediatr Neurol 2013;48: $115-122$.

18 Jayakar PB, Seshia SS: Electrical status epilepticus during slow-wave sleep: a review. J Clin Neurophysiol 1991;8:299-311.

19 Kramer U, Sagi L, Goldberg-Stern H, Zelnik N, Nissenkorn A, Ben-Zeev B: Clinical spectrum and medical treatment of children with electrical status epilepticus in sleep (ESES). Epilepsia 2009;50:1517-1524.

20 Das JC: Electroencephalogram (EEG) in the management of epilepsy in children. Mymensingh Med J 2014;23:406-411. 